\title{
PROGRAMA DE ORIENTAÇÃO E CUIDADO A GESTANTES ADOLESCENTES DE BAIXO RISCO
}

\author{
Ana Márcia Spanó Nakano*, Flávia Azevedo Gomes**, Marta Angélica Iossi Silva**, \\ Ana Cristina Magazoni Bragheto***, Ana Maria Pimenta Carvalho****
}

\section{RESUMO}

A experiência aqui relatada se refere ao projeto de extensão à comunidade, intitulado Programa de orientação a gestantes adolescentes de baixo risco, financiado pelo Fomento às Iniciativas de Cultura e Extensão (antigo Fundo de Cultura e Extensão Universitária) da Pró-Reitoria de Cultura e Extensão Universitária, da Universidade de São Paulo e desenvolvido em uma Maternidade do Município de Ribeirão Preto.

O público-alvo foram gestantes adolescentes, independente do período gestacional, que eram convidadas a participar espontaneamente das atividades propostas. Este programa teve como objetivos favorecer a adaptação da adolescente à maternidade; auxiliar no desenvolvimento de capacidades para prestar cuidado ao filho; favorecer o autocuidado no puerpério; incentivar o aleitamento materno exclusivo até o 60 mês de vida e a sua continuidade juntamente com a alimentação complementar até dois anos, além de possibilitar a oferta de um ambiente de desenvolvimento satisfatório para a criança.

Dos resultados evidenciados, verificou-se uma crescente adesão das adolescentes ao programa, além de maior envolvimento destas com o processo da maternidade e no preparo psicoemocional para o trabalho de parto e parto. Observamos também, um envolvimento satisfatório no cuidado do recém-nascido no período de internação no alojamento conjunto. Frente aos bons resultados apresentados, a instituição passou a implementar tais ações, oferecendo regularmente o programa que se constitui de uma importante estratégia na busca da assistência integral e humanizada à gestante adolescente e a sua família.

Palavras-chave: Gravidez. Adolescência. Enfermagem. Educação em saúde.

\section{ABSTRACT}

This report refers to the community service project entitled Program for teenager pregnancy of low risk, sponsored by Fomento às Iniciativas de Cultura e Extensão (antigo Fundo de Cultura e Extensão Universitária) da Pró-Reitoria de Cultura e Extensão da Universidade de São Paulo and developed in a maternal care unit in Ribeirão Preto.

Teenager pregnant girls, at any time of pregnancy, were invited to participate of meetings that had the aim of promoting healthy coping strategies regarding pregnancy and motherhood; developing skills for caring the baby and self care; promoting breastfeeding until the child completed six month and its continuity as food complement until the child was two years old as well as offering opportunity for discussing about the construction of a healthy environment for raising a child.

Results showed a growing participation in those meetings as well as a commitment with motherhood and labor/delivery. It was also verified good performance in skills required for caring the baby during the stay in rooming in. Because of the good results of this program the institution included it in routine of assistance to teenager pregnant girls and their families.

Key words: Pregnancy. Adolescence. Nursing. Health education.

\footnotetext{
* Professora Associada do Departamento de Enfermagem Materno-Infantil e Saúde Pública, Escola de Enfermagem de Ribeirão Preto -USP. **Professora Doutora do Departamento de Enfermagem Materno-Infantil e Saúde Pública, Escola de Enfermagem de Ribeirão Preto-USP. ***Psicóloga, Doutoranda do Programa de Pós-Graduação em Enfermagem Psiquiátrica do Departamento de Enfermagem Psiquiátrica e Ciências Humanas, Escola de Enfermagem de Ribeirão Preto-USP, na vigência do projeto. ****Professora Doutora do Departamento de Enfermagem Psiquiátrica e Ciências Humanas, Escola de Enfermagem de Ribeirão Preto-USP.
} 


\section{INTRODUÇÃO}

Nossa aproximação com o tema gravidez na adolescência, no cenário da prática profissional, colocou-nos frente à realidade de ser este um fenômeno tradicionalmente concebido como um problema de saúde pública, que expõe a adolescente a risco biológico e social. Independente dos possíveis riscos apontados, a maternidade pode ter outro caráter para as adolescentes, ou seja, como fator importante no seu desenvolvimento pessoal e social. O olhar para estas questões tem nos conduzido ao desafio de melhor compreender as adolescentes, em suas diferentes vivências e espaços sociais e desenvolver ações de cuidado a este grupo específico.

A adolescência é uma etapa da vida caracterizada por um complexo processo de desenvolvimento biológico, psicológico e social. Além disso, um conjunto de experiências marca a vida do adolescente: o desenvolvimento do autoconhecimento que dá origem aos sentimentos de auto-estima e de questionamento dos valores dos pais e dos adultos em geral; os impulsos sexuais ganham uma expressão mais efetiva em função da maturidade física, e a percepção do início da potencialidade da procriação (RUZANY, 2000).

Pesquisas relacionadas à gestação e à maternidade na adolescência têm mostrado alta prevalência de iniciação sexual precoce e pouca utilização de métodos contraceptivos, levando ao aumento da incidência de gestação e de doenças de transmissão sexual entre adolescentes, sobretudo entre as mais jovens (CHABON et al., 2000).

Dados no Brasil demonstram que apesar do número de gestações na adolescência estar decrescendo entre adolescentes mais velhas, este número tem se mantido elevado na faixa etária de 10 a 14 anos (BRASIL, 2006; GAMA et al., 2002; SABROZA et al., 2004).

Segundo o Ministério da Saúde, a proporção de gravidez entre 15 e 19 anos nos índices de fecundidade aumentou, paralelamente à diminuição da proporção entre as demais faixas etárias. Dados indicam que, atualmente, $37 \%$ do total de partos realizados em instituições do Sistema Único de Saúde (SUS) são de mulheres na faixa etária dos 10 aos 19 anos (BRASIL, 2006).

Com relação ao número de nascidos vivos, o Brasil apresentou em 2005, 661.137 nascimentos de crianças, cujas mães estavam na faixa etária de 10 a 19 anos, correspondendo a $21,78 \%$ do total de nascidos vivos no país. No mesmo ano, o Estado de São Paulo registrou 104.919 nascidos vivos para a mesma faixa etária de mães, ou seja, 16,95\% do total de nascidos vivos (BRASIL, 2009).

Em Ribeirão Preto-SP os dados não diferem do panorama nacional e estadual. No município, houve, em 2007, 947 nascidos vivos de mães menores de 19 anos, residentes no município, o que correspondeu a 13,08\% do total de nascidos vivos para o mesmo ano (RIBEIRÃo PRETO, 2009). 0 município também apresenta uma alta e crescente taxa de gestações na adolescência, principalmente entre as mais jovens (RIBEIRO et al., 2000). Como podemos observar na faixa etária menor de 14 anos o município apresentou em 2005 o correspondente a $0,46 \%$ do total de nascidos vivos, em 2006 verificou-se 0,57\% e em 2007 este índice subiu para 0,61\% do total de nascidos vivos (RIBEIRÃO PRETO, 2009).

Um dos aspectos que tem chamado a atenção para este grupo específico é o fato de que, para a adolescente, o pré-natal, particularmente "a consulta inicial gera grandes expectativas, que vem carregada de dúvida, culpa, vergonha, temores em relação a sua capacidade reprodutiva e desconfiança de como será o atendimento pelo profissional” (BRASIL, 1993; FERREIRA, 2006).

Para a adesão da adolescente ao espaço que lhe é oferecido, é necessário permitir que ela seja ouvida, possa expor suas ideias, sentimentos e experiências, e que também seja respeitada e valorizada (JEOLAS, 2003). Exige-se, portanto, que os serviços de saúde tenham um enfoque com base na especificidade deste grupo e na integralidade da atenção à saúde, não apenas nos aspectos técnico e biológico, mas também nos aspectos psicossociais, históricos, sociais, culturais, políticos, nos valores e comportamentos.

Observamos que a maternidade na adolescência tem merecido atenção especial nos últimos tempos em razão do número expressivo de sua ocorrência em nossa sociedade. Ao analisar a gravidez na adolescência busca-se identificar pontos críticos desta situação para a criação de alternativas ou possibilidades para compreendê-la e minimizar as repercussões psicossociais que uma gravidez pode desencadear para adolescente. Qualquer que seja a idade na gravidez, a mulher passa por uma crise situacional, decorrente da perspectiva de mudança de papel social, necessidade de adaptação, reajustes interpessoais e pela mudança de identidade. 
A falta de recurso social contribui para os problemas de saúde nas adolescentes grávidas e das mães jovens. A sobrecarga de responsabilidade, decorrente da chegada de um filho, ocasiona modificação radical no estilo de vida das adolescentes. Além disso, a falta de aceitação da gravidez pela família e pela comunidade pode determinar sérias consequências de ordem psicológica e social (YAZLLE et al., 2002).

Frente a esta realidade é que nos propusemos desenvolver um Programa de Orientação a gestantes adolescentes de baixo risco, atendidas na "Maternidade do Complexo Aeroporto - MATER”, no município de Ribeirão Preto, no sentido de direcionar esforços e uma melhor adequação do modelo assistencial no atendimento da singularidade da adolescente. Acreditamos que o desenvolvimento desta atividade de extensão à comunidade, se constitui em uma importante estratégia tanto no sentido de dar voz a este grupo, quanto potencializar a formação do profissional enfermeiro enquanto educador e agente de transformação social. Ainda cabe-nos salientar que o trabalho de extensão à comunidade além de instrumentalizador do processo dialético de teoria-prática, favorece a visão integrada do social, a democratização do conhecimento acadêmico e a participação efetiva da Universidade na comunidade, interligando as atividades de ensino com as demandas da sociedade.

O objetivo do presente artigo é relatar nossa experiência com grupos educativos para adolescentes grávidas usuárias da "Maternidade do Complexo Aeroporto - MATER”, no município de Ribeirão Preto-SP. Acreditamos que a divulgação destas ações possa subsidiar novos trabalhos em diferentes contextos para 0 alcance de uma assistência integral e humanizada à gestante adolescente e família.

\section{MATERIAIS E MÉTODOS}

Como parte do convênio celebrado entre a Universidade de São Paulo, com a interveniência da Escola de Enfermagem de Ribeirão Preto, e a Fundação Sinhá Junqueira - mantenedora da MATER, temos desenvolvido atividades de ensino, pesquisa e extensão à comunidade na atenção à mulher e família na gestação, parto, puerpério e ao recém-nascido.

Especificamente com relação às atividades de extensão à comunidade, implementamos o Programa de Orientação a gestantes adolescentes de baixo risco, desenvolvido por meio de grupos educativos. 0 referido projeto teve subsídio financeiro do Fomento às Iniciativas de Cultura e Extensão (antigo Fundo de Cultura e Extensão Universitária) da Pró-Reitoria de Cultura e Extensão Universitária da Universidade de São Paulo, no período de 2006 a 2008.

Este programa objetivou favorecer a adaptação da adolescente à maternidade; auxiliar no desenvolvimento de capacidades para prestar cuidado ao filho; favorecer o autocuidado no puerpério; incentivar o aleitamento materno exclusivo até o 6 - mês de vida e a sua continuidade juntamente com a alimentação complementar até dois anos e possibilitar a oferta de um ambiente de desenvolvimento satisfatório para a criança.

Os grupos educativos ora implementados basearam-se na concepção da educação em saúde, enquanto um campo de prática e de conhecimento da área da saúde, que se tem ocupado com a criação de vínculos, saberes e dimensões complementares entre a ação de saúde, o pensar e o fazer cotidiano (VASCONCELOS, 2006). Buscamos, portanto, uma proposta que possibilitou por meio de uma metodologia participativa e lúdica, perspectiva metodológica esta que vai além da capacidade de gerar informações, mas que considera, sobretudo, os processos sociais e culturais que ajudam a adolescente a dar sentido aos seus valores, a seus desejos, sentimentos e interesses, fortalecendo a autoestima, a autonomia, o envolvimento das emoções e sentimentos junto às cognições e, consequentemente, a resignificação e decodificação das informações (FERREIRA, 2006; SILVA, 2002).

Neste sentido, esta proposta metodológica de educação preventiva e participativa, por estabelecer uma abertura inicial entre o dito e o não-dito, possibilita a convergência dos aspectos emocionais e racionais, de forma livre e descontraída, através de um diálogo aberto, de uma linguagem simples, comum e concisa. Possibilita ainda o encontro de soluções para os desafios, explorando as possibilidades, o conhecimento mútuo, generalizando as conquistas e o aprendizado para 0 âmbito familiar e social da adolescente.

Para atingir os objetivos foram realizados encontros com as gestantes adolescentes, independente da idade gestacional, sendo aproximadamente 10 gestantes por grupo. As gestantes adolescentes usuárias do serviço de pré-natal da MATER eram convidadas a participar dos grupos juntamente com um acompanhante 
de sua escolha. A busca de integrar a família da adolescente - na figura de uma acompanhante de sua escolha - se apoia na possibilidade de ajudar a adolescente a tomar consciência de sua realidade, sentir-se segura para tomar decisões e atuar nas diferentes situações apresentadas no processo da maternidade.

Uma estratégia por nós adotada, a fim de facilitar a adesão à atividade, foi conciliar a realização do grupo com a vinda da adolescente para a sua consulta de pré-natal de rotina. O Programa de Orientação foi configurado sob a forma de oficina, com cinco encontros, uma vez por semana e duração de 1 hora e meia. A cada cinco semanas, portanto, nova oficina era iniciada o que permitia a inclusão sempre que necessário de novas participantes. Os profissionais envolvidos eram do serviço que incluem enfermeiras obstétricas, enfermeiras generalistas e docentes da Escola de Enfermagem, que se revezam de acordo com os temas no decorrer das oficinas. O grupo educativo também contou com a participação de alunos bolsistas de graduação e pós-graduação (fisioterapeutas e psicólogas), além de voluntários.

A programação dos temas desenvolvidos nos encontros se constituía em:

\section{ENCONTRO}

\section{PERCEPÇÃO CORPORAL}

Realizado por enfermeiras e fisioterapeutas, o que se buscou trabalhar foi como as adolescentes percebiam as mudanças físicas e emocionais decorrentes da gestação, utilizando de dinâmicas de grupo, técnicas de percepção corporal e exercícios para alívio dos desconfortos específicos da gravidez (lombalgia, edemas, varizes, entre outros). As mudanças percebidas eram discutidas entre as participantes do grupo com o suporte de informação do profissional de saúde que coordenava as oficinas.

\section{ENCONTRO}

\section{VÍNCULO}

Neste encontro em específico, coordenado por uma psicóloga e uma enfermeira, o objetivo era trabalhar com as adolescentes gestantes questões relacionadas a sua relação com seu futuro bebê, pontuando a relação mãe-bebê como algo que pudesse ser desenvolvido por elas, a fim de propiciar bom desenvolvimento físico e mental a esta futura mãe e seu bebê. Foram trabalhadas também as dificuldades das adolescentes no que se refere aos cuidados do bebê, como por exemplo, medo e ou receio em dar banho, cuidar de umbigo, amamentar, entre outras coisas trazidas por elas, além de ajudá-las a pensar em membros da família ou da comunidade que pudessem auxiliá-las neste momento. Outro ponto elucidado no grupo foram as expectativas de cada uma em relação ao futuro; como elas pensavam em se organizar depois do nascimento do bebê, questões relacionadas a trabalho e estudos. Os grupos foram bastante proveitosos na medida em que foi possível trabalhar questões bem singulares de cada adolescente gestante, desenvolvendo recursos para que essa futura mãe desenvolvesse um bom vínculo com seu bebê e com as pessoas a sua volta.

\section{ENCONTRO}

TRABALHO DE PARTO E PARTO

Com base nos preceitos da humanização no nascimento e parto, em que se destaca o reconhecimento do valor emocional que o acompanhante tem para a parturiente, do respeito à mulher como sujeito do processo de parturição e a crítica ao excesso de intervencionismo, aos procedimentos e regras que resultaram em profundas dissociações entre os aspectos somáticos e emocionais, não satisfazendo as necessidades da mulher, do bebê e da família, é que trabalhamos com a adolescente gestante e seu acompanhante buscando identificar suas ideias e expectativas sobre o parto e o nascimento do bebê, sobre os procedimentos que poderiam ser feitos durante o trabalho de parto e parto, as possibilidades e limitações durante o trabalho de parto e parto, o que favorece a participação consciente e mais efetiva no processo, além de informações referentes ao reconhecimento de sinais e sintomas de trabalho de parto, dos procedimentos que são realizados para resolução do parto e sinais de riscos. Era estimulada a participação da mulher e respectivo acompanhante na utilização de recursos não farmacológicos de alívio da dor e de procedimentos que favorecem a progressão do trabalho de parto. Entendemos ser fundamental dar possibilidade à mulher participar efetivamente no seu trabalho de parto e parto, resgatando a sua condição de protagonista da cena do parto.

\section{4ㅇ ENCONTRO}

ALEITAMENTO MATERNO E CUIDADO COM O RECÉM-NASCIDO

Partindo do conhecimento e experiências pessoais e familiares é que introduzimos e discutimos os referidos 
temas. Procuramos informar sobre as vantagens do aleitamento materno precoce, exclusivo, sob livre demanda e continuado e como o leite materno é produzido, além de escutar as preocupações e dúvidas em relação à amamentação, de modo a ajudar no manejo e alertar quanto aos riscos do uso de mamadeiras e chupetas. Os aspectos referentes aos cuidados com o bebê envolvem inicialmente um processo de aprendizado em relação a conhecer e identificar as necessidades do recém-nascido, implica em observar a linguagem corporal da criança, de suas manifestações de comportamento, e lidar com possíveis inseguranças para o exercício dos papéis materno de modo a integrar de forma satisfatória ao seus projetos de vidas.

\section{ENCONTRO}

\section{PUERPÉRIO E CONTRACEPÇÃO}

A grande ênfase neste momento é chamar a atenção para o auto-cuidado no puerpério. 0 cuidado no pós-parto envolve certos mitos e tabus transmitidos de geração a geração, que são trabalhados de forma a levar as adolescentes a refletir sobre a veracidade destas crenças e possibilitar a uma escolha segura, consciente e responsável do cuidado a sua saúde. Dentre as informações que se busca apresentar inclui a noção de planejamento familiar, partindo das próprias experiências de gravidez precoce e de como as adolescentes vivenciam a sua sexualidade, como se utilizam de medidas contraceptivas e sua postura frente o sexo seguro.

\section{RESULTADOS}

Esta experiência que surgiu como proposta de atividade de cultura e extensão universitária, sob coordenação de docentes e enfermeiros da Escola de Enfermagem de Ribeirão Preto-USP, com participação de alunos da graduação e pós-graduação, hoje se insere no conjunto das ações de cuidado às adolescentes gestantes que a Maternidade MATER oferece a sua clientela.

Tal proposta, ao longo destes anos, resultou na consolidação de ações tanto no serviço quanto na comunidade, visto que se observa um aumento na participação de gestantes adolescentes no programa, seja por meio da busca espontânea ou encaminhada por serviços de saúde e equipamentos sociais do município. No ano de 2005, quando implementada a proposta, participaram do curso de gestantes 105 adolescentes, obtendo um aumento significativo de participantes no ano de 2006, 274 participantes. Em 2007 tivemos 237 gestantes participantes e de janeiro a agosto de 2008 tivemos 206 participantes.

Cabe destacar que em 2008, em um semestre, o número de participantes praticamente atinge o valor total de participação no ano de 2007. Isto reflete a repercussão positiva desta atividade entre as adolescentes que divulgam o curso em seu meio social (comunidade, família, escola).

A implementação e oferecimento regular das ações relacionadas ao Programa de Orientação a Gestantes Adolescentes de Baixo Risco resultaram na reorganização do atendimento em outros setores da maternidade, destacando-se a implementação da consulta para elaboração do plano de parto para as gestantes adolescentes. Como prioridade e buscando otimizar o atendimento, fixou-se um dia da semana somente para $o$ atendimento das gestantes adolescentes, de modo a otimizar a ida destas à maternidade, para o pré-natal, e assim participar do programa de orientação. A especificidade dada ao cuidado das gestantes adolescentes se estendeu para o todo cuidado no ciclo gravídico puerperal (na resolução do parto e no alojamento conjunto). Numa perspectiva futura, está prevista a ampliação dessas ações na assistência ambulatorial à gestante adolescente como parte das metas propostas à Secretaria da Saúde do Estado de São Paulo, que em 2009 passa a incorporar a MATER e transformá-la em um Centro de Referência para Saúde da Mulher.

\section{DISCUSSÃO}

Analisando como as ações tem se refletido para as adolescentes, observamos um maior envolvimento das adolescentes gestantes com o processo da maternidade, em termos de estarem mais preparadas para 0 trabalho de parto e parto, no cuidado do recém-nascido e consigo mesma. Ampliou-se a participação de membros da família para receber orientação e participar do cuidado durante a fase de internação, configurandose em uma real interseção de apoio afetivo-material (DIAS \& AQUINO, 2006).

A integração da família da adolescente - na figura do acompanhante de sua escolha - no processo 
de parturição e no pós-parto é uma estratégia que se mostra efetiva para que a adolescente tome consciência de sua realidade, sinta-se segura para tomar decisões e atuar nas diferentes situações apresentadas.

Diferentes estudos têm apontado controvérsias quanto à compreensão da gravidez na adolescência como um problema e risco atribuídos pela construção negativa deste fenômeno social, demonstrando que, muitas vezes, este se constitui em uma possibilidade de busca da autonomia e responsabilidade, de crescimento e amadurecimento pessoal, no desejo consciente de ser mãe ou pai, e até mesmo em uma fonte de satisfação (PANTOJA, 2003; GONTIJO \& MEDEIROS, 2008; FOLLE \& GEIB, 2004).

Corroboramos com a ideia de Ayres \& Junior (2000, p.81) de que existe uma urgente necessidade de evitarmos estereótipos "que nos fazem ver a gestante adolescente como mais uma vítima infeliz de um ato inconsequente que lhe roubará os anos dourados da juventude". Ainda segundo estes autores, a interpretação limitada, estereotipada e preconceituosa desse fenômeno por parte dos serviços e profissionais, pode trazer consequências danosas, em termos humanísticos, mais do que qualquer "risco gravídico".

As novas responsabilidades assumidas pela adolescente e o potencial amadurecimento pessoal são fatores que podem estimular, inclusive, um maior envolvimento e melhor relacionamento no cuidado ao recémnascido, caracterizado e percebido em muitas famílias como atencioso, cuidadoso, atendendo plenamente às necessidades básicas da criança (MACHADO et al., 2003; FOLLE \& GEIB, 2004).

Para os profissionais, a implementação desse programa contribuiu na formação de enfermeiras obstétricas do Programa de Especialização de Enfermagem Obstétrica e Neonatal, do Departamento de Enfermagem Materno-Infantil e Saúde Pública da EERP-USP e de estudantes do curso de Bacharel em Enfermagem, pois puderam vivenciar experiências e aprendizagens significativas que possibilitaram sua formação enquanto agentes de transformação para um cuidado integral.

A participação dos bolsistas de graduação e de pós-graduação, ou mesmo de voluntários neste Programa de orientação, foi outra iniciativa que resultou no aprimoramento técnico e troca de experiências, tanto durante as atividades em grupo, quanto em reuniões entre a equipe executora, essencial à formação de uma visão multidisciplinar.
Os profissionais habilitados no manejo dos aspectos clínicos, emocionais e sociais quando incluídos na assistência à adolescente gestante, como neste trabalho, trazem muitos ganhos para a saúde do binômio mãe-filho, tanto em projetos de prevenção e educação em saúde, quanto no atendimento desta população específica na rede assistencial, visando deste modo, a promoção de uma melhor qualidade de vida.

A perspectiva deste trabalho multiprofissional e interdisciplinar veio atender ao apontado pela literatura, como uma apropriada forma de responder às demandas individuais e coletivas dos adolescentes, considerando o seu contexto, o seu cotidiano e a prioridade da atenção integral e humanizada (FORMIGLI et al., 2000).

Ao idealizarmos este programa, procuramos trabalhar a integralidade da atenção à gestante e posteriormente à puérpera adolescente, tanto na perspectiva da integralidade focalizada e da integralidade ampliada. A primeira que resulta do esforço de cada um dos trabalhadores e da equipe como um todo. Deste modo, o atendimento de cada profissional é realizado na perspectiva de que a integralidade pretendida só será alcançada como fruto do trabalho da equipe de saúde, com seus múltiplos saberes e práticas. A segunda, ou seja, a integralidade ampliada, deve ser pensada e estabelecida a partir da articulação entre os serviços de saúde e entre estes e outros setores e instituições sociais (CECÍLIO, 2001).

Frente ao exposto, destacamos que um importante avanço a ser considerado no programa aqui apresentado e no contexto do cuidado implementado, é os profissionais se mostraram mais abertos a apreender as singularidades das vivências maternas e necessidades das adolescentes, readequando o modelo assistencial, com vistas a uma atenção mais humanizada.

\section{CONCLUSÕES}

Com base no projeto realizado, pudemos concluir que a realização de grupos com adolescente gestantes é de extrema importância na atenção ao pré-natal, em suas diferentes dimensões, além de contribuir para que as adolescentes se sintam mais seguras e tranquilas no momento do parto e nos cuidados pós-parto, abrindo possibilidades de torná-las protagonistas das vivências da maternidade. 
Acreditamos, ainda, que oferecer oportunidades a estas adolescentes para reflexão e aquisição de habilidades e competências é sem dúvida potencializar hábitos de vida mais saudáveis e novas perspectivas de vida, prevenindo e minimizando possíveis problemas que possam vir a encontrar neste momento singular de vida.

Por meio deste programa, percebemos ainda que os grupos de educação em saúde têm propiciado uma maior participação da família na vida da mãe adolescente, abrindo um espaço até então inexistente, sendo as dificuldades e os constrangimentos superados, até mesmo pela forma com que esta passa a ver e se relacionar com a adolescente. Podemos destacar que os grupos realizados, as orientações e reflexões oportunizadas estão influenciando de maneira positiva o pensar e repensar da vida cotidiana e futura de cada adolescente, onde o comportamento preventivo tem sido a ênfase desta possibilidade.

Outro aspecto a ser destacado centra-se no fato de atender a prerrogativa da Universidade desenvolver atividades de retorno ao contexto social externo a seus muros.

Neste sentido, o programa aqui relatado se constitui, sem dúvida, em uma iniciativa que colabora e possibilita a efetivação de serviços ancorados nos princípios da integralidade, universalidade, equidade e humanização, ou seja, na efetiva luta de visibilidade e implementação do Sistema Único de Saúde no Brasil.

\section{REFERÊNCIAS BIBLIOGRÁFICAS}

AYRES, J.R.C.M.; JUNIOR. I.F. Saúde do Adolescente. In: SCHARAIBER, L.B, NEMES, M. I. B., MENDES-GONÇALVES, R.B (Org.). Saúde do adulto: programas e ações na unidade básica. São Paulo: Hucitec, 2000, p. 66-85. (Saúde em Debate, 96, Série Didática; 3 ).

BRASIL. Departamento de Informática do SUS. Estatísticas Vitais - Mortalidade e Nascidos Vivos. Disponível em: $<$ http://tabnet.datasus.gov.br/cgi/tabcgi.exe?sinasc/cnv/ nvsp.def>. Acesso em: 04 abr. 2009.

BRASIL. Ministério da Saúde. Marco teórico e referencial: saúde sexual e saúde reprodutiva de adolescentes e jovens. Brasília: Ministério da Saúde, 2006. 56 p. (Série B. Textos Básicos de Saúde).

BRASIL. Ministério da Saúde. Secretaria de Assistência à Saúde. Departamento de Assistência e Promoção à Saúde. Serviço de Assistência à Saúde do Adolescente-SASAD. Normas de atenção à saúde integral de adolescente. Vol. 3
- Assistência ao Pré-Natal, ao Parto e ao Puerpério - Planejamento Familiar — Doenças Sexualmente Transmissíveis - Problemas Ginecológicos. Brasília: Ministério da Saúde, 1993.

CECÍLIO, L.C.O. As necessidades de saúde como conceito estruturante na luta pela integralidade e equidade na atenção em saúde. In: PINHEIRO, R.; MATTOS, R.A. (Org.). Os sentidos da integralidade na atenção e no cuidado à saúde. Rio de Janeiro: UERJ: ABRASCO, 2001, p. 113-126.

CHABON, B.; FUTTERMAN, D.; HOFFMAN, N.D. HIV and AIDS in adolescents. Pediatric Clin., v. 47, n. 1, p. 171-187, 2000.

DIAS, A.B.; AQUINO, E.M.L. Maternidade e paternidade na adolescência: algumas constatações em três cidades do Brasil. Cad Saúde Pública, v. 22, n. 7, p. 1447-1458, 2006.

FERREIRA, M.A. A educação em saúde na adolescência: grupos de discussão como estratégia de pesquisa e cuidadoeducação. Texto Contexto Enferm. v. 15, n. 2, p. 205-11, 2006.

FOLLE, E.; GEIB, L.T.C. Representações sociais das primíparas adolescentes sobre o cuidado materno ao recém-nascido. Rev Latino-Am Enfermagem, v. 12, n. 2, p. 183-90, 2004.

FORMIGLI, V.L.A.; COSTA, M.C.O.; PORTO, L.A. Avaliação de um serviço de atenção integral à saúde do adolescente. Cad de Saúde Pública, v. 16, n. 3, p. 177-184, 2000.

GAMA, S.G.N.; SZWARCWALD, C.L.; LEAL, M.C. Experiência de gravidez na adolescência, fatores associados e resultados perinatais entre puérperas de baixa renda. Cad Saúde Pública, v. 18, n. 1, p. 153-161, 2002.

GONTIJO, D.T.; MEDEIROS, M. Tava morta e revivi: significado de maternidade para adolescentes com experiência de vida nas ruas. Cad Saúde Pública, v. 24, n. 2, p. 469-472, 2008.

JEOLAS, L.S.; FERRARI, R.A.P. Oficinas de prevenção em um serviço de saúde para adolescentes: espaço de reflexão e de conhecimento compartilhado. Ciênc. saúde coletiva, v. 8, n. 2, p. 611-620, 2003.

MACHADO, F.N.; MEIRA, D.C.; MADEIRA, A.M.F. Percepções da família sobre a forma como a adolescente cuida do filho. Rev Esc Enfermagem USP, v. 37, n. 1, p. 11-8, 2003.

PANTOJA, A.L.N. Ser alguém na vida: uma análise sócio-antropológica da gravidez/maternidade na adolescência, em Belém do Pará, Brasil. Cad Saúde Pública, v. 19, Supl 2, p. 335-343, 2003.

RIBEIRÃO PRETO. Secretaria Municipal da Saúde. Divisão de Vigilância Epidemiológica. Dados referentes a Nascidos Vivos no Município de Ribeirão Preto. Disponível em: <http://www.ribeiraopreto.sp.gov.br/ssaude/i16principal. asp? pagina=/ssaude/vigilancia/vigep/tabnet/i16indice. htm>. Acesso em: 4 abr. 2009. 
RIBEIRO, E.R.; BARBIERI, M.A.; BETTIOL, H.; SILVA, A.A. Comparação entre duas coortes de mães adolescentes em município do Sudeste do Brasil. Rev Saúde Pública, v. 34, p. 136-142, 2000.

RUZANY, M. H. Mapa da situação de saúde do adolescente no município do Rio de Janeiro. Rio de Janeiro, 2000, 113 p. Tese, Fundação Oswaldo Cruz, Escola Nacional de Saúde Pública.

SABROZA, A.R.; LEAL, M.C.; GAMA, S.G.N.; COSTA, J.V. Perfil sociodemográfico e psicossocial de puérperas adolescentes do Município do Rio de Janeiro, Brasil - 1999-2001. Cad Saúde Pública, v. 20, Supl 1, p. 112-120, 2004.

SILVA, R.C. Metodologias participativas para trabalhos de promoção de saúde e cidadania. São Paulo: Vetor, 2002.

VASCONCELOS, E.M. Educação popular e a atenção à saúde da família. 3‥ edição. São Paulo: Hucitec, 2006. (Saúde em debate: 130).

YAZLLE, M.E.H.D.; MENDES, M.C.; PATTA; M.C.; ROCHA, J.S.Y.; AZEVEDO, G.D.; MARCOLIN, A.C. A adolescente grávida: alguns indicadores sociais. Revista Bras. Ginecol. Obstet., v. 24, n. 9, p. 609-614, 2002. 